\title{
Desarrollo de competencias informacionales en contextos universitarios: enfoques, modelos y estrategias de intervención *
}

\author{
Gloria Patricia Marciales Vivas ** \\ Jorge Winston Barbosa Chacón ***

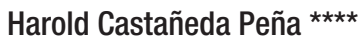

Artículo recibido:

23 de febrero de 2013.

Artículo aceptado:

11 de marzo de 2013.

\section{RESUMEN}

El desarrollo de Competencias Informacionales ocupa un lugar destacado en instituciones de educación superior en razón a la relevancia de estas competencias para la sociedad de la información. De allí que numerosas publicaciones se hayan realizado sobre esos instrumentos a través de los cuales las universidades responden a los desafíos que esto supone. En este artículo

* Artículo resultado del proyecto de investigación titulado "Eventos atribuidos como significativos en la reconfiguración de la competencia informacional por estudiantes universitarios". Proyecto respaldado por la Pontificia Universidad Javeriana, Bogotá-Colombia, Cód. PS4663-Vicerrectoría Académica, y la Universidad Industrial de Santander, Bucaramanga-Colombia, Cód. CH 2012-2-Vicerrectoría de Investigación y Extensión.

**Pontificia Universidad Javeriana, Colombia. gloria.marciales@javeriana.edu.co

*** Universidad Industrial de Santander, Colombia. jowins@uis.edu.co

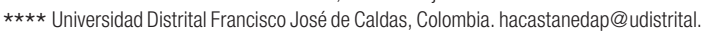
edu.co

INVESTIGACIÓN BIBLIOTECOLÓGICA, Vol. 29, Núm.65, enero/abril, 2015, México, ISSN: 0187-358X. pp. 39-72 
se presenta una revisión documental correspondiente a los últimos siete años de publicaciones en las cuales se reportaron acciones dirigidas al desarrollo de competencias informacionales en jóvenes universitarios. Se emplearon cuatro bases de datos, más un repositorio de publicaciones de Alfabetización Informacional en Iberoamérica. Los artículos se clasificaron teniendo en cuenta tres categorías: enfoques, modelos y estrategias. Los enfoques revelan el predominio de perspectivas cognitivistas y una incipiente incorporación de la perspectiva sociocultural. Los modelos identificados dan cuenta de logros valiosos así como de dificultades de orden institucional, económico y administrativo que limitan su implementación. Las estrategias se presentan desvinculadas de propuestas en las cuales se integren agentes educativos, situación que afecta su impacto. La principal falencia de los estudios revisados son los instrumentos de evaluación para dar cuenta de los resultados obtenidos. Para terminar se formulan preguntas y retos para las ciencias de la información.

Palabras clave: Alfabetización informacional; Competencias informacionales; Estudiantes universitarios; Enfoques teóricos; Modelos de desarrollo de la competencia informacional; Estrategias de intervención.

\section{Abstract}

Development of information skills in college: approaches, models and intervention strategies Gloria-Patricia Marciales-Vivas, Jorge-Winston BarbosaChacón and Harold Castañeda-Peña

Due to its significance in the information society, information literacy must be stressed in institutions of higher education. There is a wealth of research on the instruments employed by such institutions to keep up to date with new challenges regarding information literacy. This study provides a review of the last seven years of literature reporting on information literacy teaching strategies for university students contained in four databases and a single web-portal. Journal articles were classified into three main thematic categories: approaches, models and strategies. The review reveals that the category of approaches is largely dominated by 
cognitive perspectives at the expense of sociocultural methods. The models identified reflect considerable achievements, but also institutional, economic and administrative burdens that hinder implementation. Strategies appear to be disassociated from proposals entailing educational agents, a situation that undermines their impact. The central weakness of the studies reviewed lies in the assessment instruments used to yield results. The paper concludes posing questions and challenges for field of information science.

Keywords: Information Literacy; Information Skills; College Students; Theoretical Approaches; Models of Information Skills Development; Intervention Strategies.

\section{INTRODUCCIÓN}

—1 lobjetivo de este artículo es describir algunos de los enfoques, modelos Ede intervención y estrategias reportados en publicaciones sobre investigación y experiencias, dirigidos al desarrollo de competencias informacionales en jóvenes universitarios. Se seleccionaron publicaciones en revistas indizadas en un periodo de tiempo de siete años (2007-2013). Se estableció este periodo teniendo en cuenta que, además de ser un rango de actualidad, la revisión hace parte de un proyecto de investigación, dirigido por los autores de este artículo, sobre los eventos que los estudiantes universitarios identifican en su formación profesional como significativos por su impacto en el desarrollo de competencias informacionales. Los estudiantes que participaron en dicho estudio corresponden a la cohorte que inició en 2009; experiencia de formación que resulta en cierta forma paralela a las publicaciones que tuvieron avances en este campo de problemas.

Para esta revisión se emplearon cuatro bases de datos: Sage Journals, Scopus, ISIWeb y ProQuest. Las bases de datos fueron elegidas teniendo en cuenta: i) el nivel de reconocimiento científico y su clasificación en bases de datos con categoría "Multidisciplinarias" y "Ciencias Sociales y Humanas"; clasificación en donde se ubican las competencias informacionales como objeto de estudio y ii) un repositorio reconocido en torno a publicaciones sobre Alfabetización Informacional en Iberoamérica. Los artículos corresponden a investigaciones y experiencias en educación superior publicadas en revistas con reconocimiento internacional. 
El concepto de competencia informacional adoptado por los investigadores y académicos de los artículos no es discutido aquí, así como tampoco los instrumentos de evaluación empleados en el caso de las investigaciones. Solamente se hace una descripción de los enfoques, modelos y estrategias que marcan el derrotero de las publicaciones en este campo de problemas, específicamente en contextos universitarios.

Si bien algunos de los artículos incluyen los tres elementos estudiados (enfoque, modelos, estrategias), se identificó aquello que constituía el eje articulador del trabajo realizado de manera que aparece en más de una de las tres categorías elegidas.

\section{Metodología}

Se llevó a cabo una revisión de literatura en cuatro bases de datos (Sage Journals, Scopus, ISIWeb, ProQuest) y en el repositorio web sobre alfabetización en Iberoamérica, el cual es liderado por Alejandro Uribe Tirado y en donde se agrupan referencias de libros, artículos, ponencias y eventos propios de países iberoamericanos. ${ }^{1}$ Se utilizaron como palabras clave en inglés y español las siguientes: information literacy/competencia informacional, university students/estudiantes universitarios, higher education/educación superior e information literacy skills/habilidades de alfabetización informacional. Los artículos se clasificaron teniendo en cuenta que cumplieran con al menos una de las tres categorías elegidas: enfoques teóricos, modelos de intervención y estrategias de alfabetización. Se seleccionaron artículos publicados en idioma inglés y español entre los años 2007-2013 en revistas indizadas. Se identificaron 50 artículos que cumplían con alguna de las tres categorías seleccionadas, 27 artículos de investigación y 23 artículos sobre experiencias de implementación de programas (Ver Anexo). Al respecto, en los Gráficos 1 y 2 se puede apreciar una descripción de la literatura encontrada según su distribución.

1 Alfabetización Informacional en Iberoamérica-Estado del Arte. El objetivo de este Wiki-Repositorio es construir de una manera colaborativa un estado del arte sobre la Alfabetización Informacional en Iberoamérica. Consultar: http://alfiniberoamerica.wikispaces.com/ 


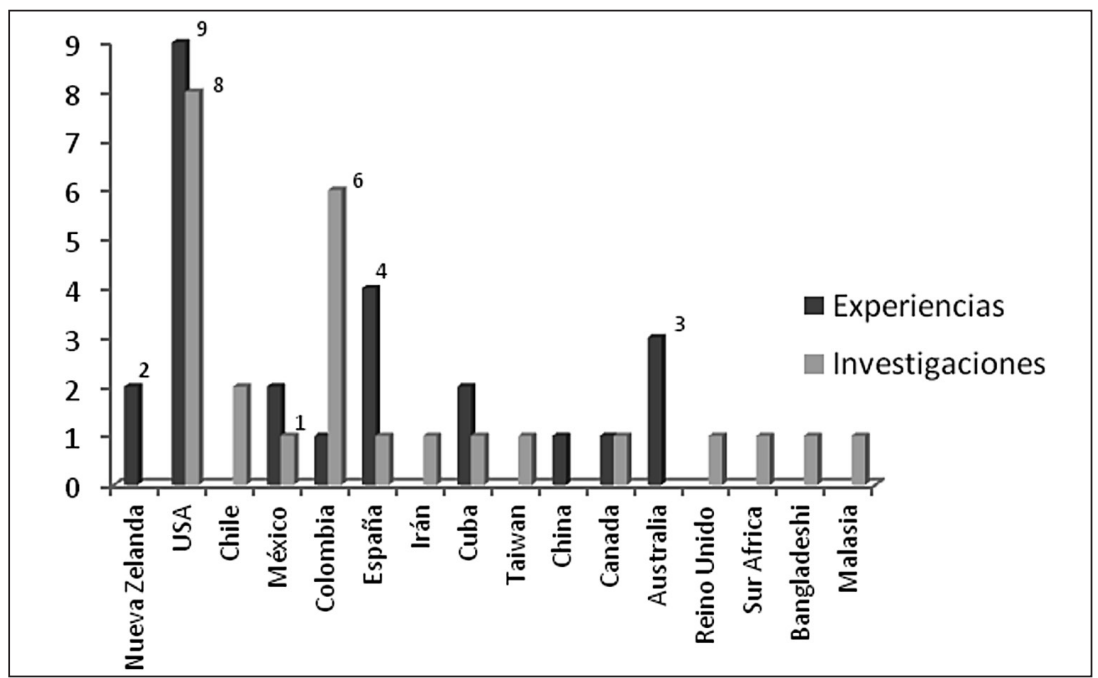

Gráfico 1. Distribución de publicaciones (Experiencias e Investigaciones) por países.

Fuente: Elaboración propia

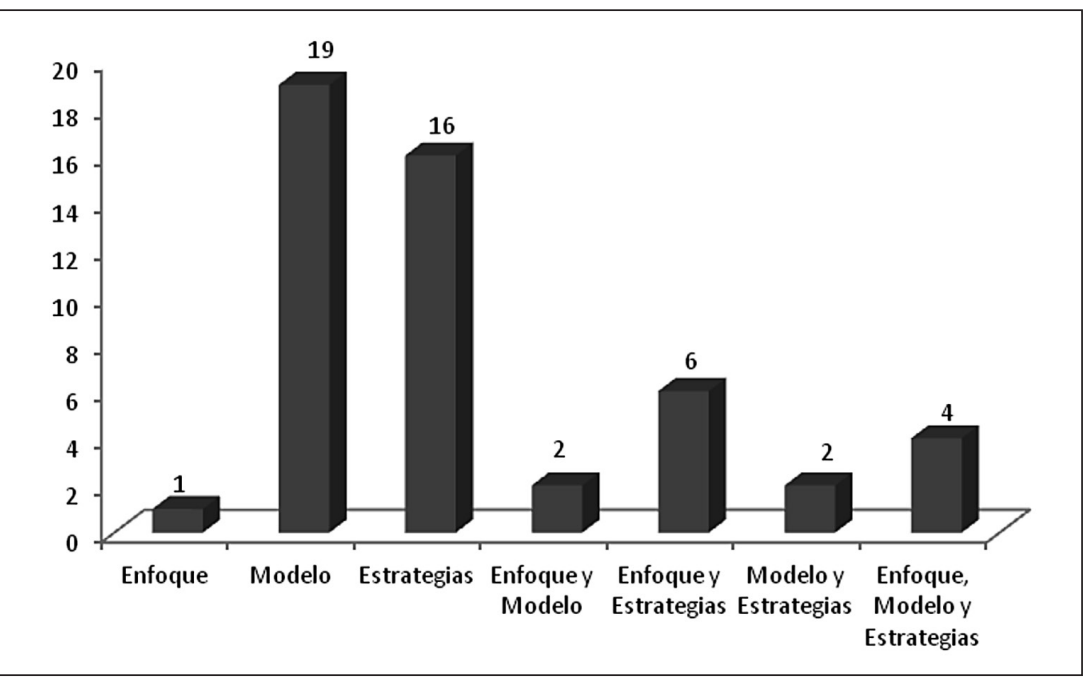

Gráfico 2. Distribución de las publicaciones según la presencia de los elementos (Enfoque, Modelo y Estrategias). Fuente: Elaboración propia

A continuación se describen los hallazgos correspondientes a cada categoría. 


\section{Enfoques Teóricos en el Desarrollo de Competencias Informacionales}

El enfoque se entiende en este artículo como el conjunto de referentes epistemológicos y teóricos que sustentan la intervención que se lleva a cabo para el desarrollo de competencias informacionales. La revisión de la historia de la Ciencia de la Información en lo que respecta a este campo de problemas permite identificar los diversos enfoques que han orientado las acciones dirigidas al desarrollo de estas competencias: objetivista, cognitivista e histórico cultural (Montiel-Overall, 2007).

La perspectiva objetivista considera que los usuarios adquieren información de fuentes reconocidas por una autoridad (Marciales Vivas et al., 2006), de manera que las acciones para el desarrollo de competencias informacionales se orientan al fortalecimiento de habilidades a través de la práctica reiterada y los resultados de estas acciones son evaluados mediante pruebas objetivas para verificar los conocimientos adquiridos (Montiel-Overall, 2007).

La segunda perspectiva, influenciada por las ideas de Dewey (1938) sobre el aprendizaje experiencial, destaca el lugar de los aprendices como pensadores activos y constructores de significados y busca desarrollar habilidades instrumentales para acceder, evaluar y usar información con sentido para los usuarios. En este escenario se ubican, con gran trascendencia, muchas de las experiencias actuales de las Ciencias de la Información y la Documentación las que, a través de programas de formación, hacen la apuesta de contribuir con el desarrollo de habilidades informativas para apoyar el aprendizaje y la adquisición de conocimientos científicos y tecnológicos y así favorecer el desarrollo académico y personal. Como referencia de ello han surgido diversas asociaciones y declaraciones a partir de las cuales se deriva un gran marco conceptual y normativo (Barbosa-Chacón et al., 2010).

La tercera perspectiva recibe influencia del pensamiento de Vygotsky (mediados de la década de los 90) e introduce el concepto de competencia informacional como práctica con dimensión social y cultural; hace énfasis en la relación entre su desarrollo y la formación de un sujeto social capaz de asumir con conciencia tanto crítica como ética la diversidad y complejidad de factores culturales que median el acceso a la información y la información misma. La actividad humana es entendida como situada en la interacción con otros, como mediadora de la construcción de conocimiento y a las diferencias culturales y contextuales como configuradoras de creencias y prácticas cotidianas. 
La revisión de la investigación y de las experiencias de desarrollo de la competencia informacional permite apreciar que la pregunta por el enfoque no es dominante en la literatura, aunque las mismas propuestas dejan vislumbrar los referentes en los cuales éstas se sustentan. Se encuentra por ejemplo que el enfoque comportamental, dominante en otras épocas, está ausente en los trabajos que se han adelantado en los últimos siete años, en tanto que el enfoque cognitivista es evidente en varios de los estudios analizados (Cortés, 2007; Loyola y Matus, 2007; Valadez et al., 2007; Cabral, 2008; Hanson y Williams, 2008; Hernández Hernández, 2010; Uribe-Tirado y Castaño, 2010; Pinto y Uribe, 2010; Rastgoo et al., 2011; Álvarez, Ríos y Velásquez, 2012). En estos últimos se da cuenta del tipo de conocimiento (declarativo, procedimental, condicional), así como de las estrategias cognitivas y metacognitivas que se ponen en juego en el manejo de información, y los cambios que a nivel cognitivo se generan como resultado de una intervención. Cabe destacar aquí el estudio de Rastgoo et al. (2011), en el cual la interacción en línea es empleada para el desarrollo de procesos metacognitivos.

El enfoque sociocultural, empleado como referente en algunos de los trabajos, sirve de base en algunas acciones cuyo propósito ha sido comprender las prácticas de las comunidades en las cuales se configuran las formas de acceder, evaluar y hacer uso de la información, para proyectar a partir de allí estrategias de intervención (Wang, L. 2006; Wang, Y. 2007; Loyola y Matus, 2007). En esta línea se encuentran también los estudios de Wang, Bruce y Hughes (2011) sustentados en tres principios básicos propios del enfoque adoptado: en el primero, el conocimiento es socialmente construido y, por tanto, la naturaleza social del desarrollo cognitivo sirve como modelo dialógico para entender cómo la competencia informacional puede ser integrada al currículo en una comunidad de práctica. En el segundo, las herramientas culturales desempeñan un papel importante en las interacciones sociales ya integradas curricularmente; y en el tercero, la apropiación alcanzada puede servir como modelo cuando los datos son reunidos y analizados con este enfoque.

En relación con el primer principio, la realidad se construye a partir de la experiencia de la gente; se afirma así que el conocimiento es una construcción entre individuos y entre miembros de un grupo. El segundo principio propone entender el aprendizaje y el desarrollo como mediados por herramientas o signos semióticos, en donde las herramientas culturales sirven para transformar la naturaleza y contribuyen a la propia transformación (Kozulin, 2000). De acuerdo con el tercer principio, el aprendizaje y el desarrollo son procesos de internalización que van de lo interpersonal a lo intrapersonal en un proceso de construcción de sentido. 
Cabe señalar que, si bien algunas de las investigaciones y experiencias revisadas utilizan el término cultura para hacer referencia a las prácticas de uso de fuentes de información, pocas hacen realmente un análisis de las expresiones culturales que se actualizan en tales prácticas. El término cultura por tanto es empleado en un sentido débil para denominar la existencia de prácticas comunes en grupos humanos sin profundizar en éstas con una perspectiva sociocultural.

Lo anterior sugiere que éste es un camino investigativo que habría que fortalecer y que permitiría comprender, por ejemplo, las prácticas de uso de fuentes de información de los jóvenes como usuarios de información en aquellos contextos que les son propios, sin caer en generalizaciones como las de "nativos digitales", ni en descalificaciones que sugieran que las nuevas generaciones carecen de aquellas competencias con las que sí cuentan las anteriores. Epistemológicamente supondría reconocer el carácter construido del conocimiento en procesos de interacción social, de manera que el desarrollo de competencias informacionales requeriría la adopción de un modelo dialógico integrado al currículo coherente con las culturas disciplinares.

\section{Modelos de Intervención para el Desarrollo de Competencias Informacionales}

Un modelo es una representación de los elementos estructurales y funcionales que constituyen un fenómeno complejo, así como de las relaciones existentes entre éstos, con lo cual contribuyen a la comprensión de dichos fenómenos. Como estructura conceptual esto sugiere un marco de ideas para un conjunto de descripciones que difícilmente podrían ser sistematizadas (Hanson, 1958).

En lo que respecta a las prácticas dirigidas al desarrollo de competencias informacionales éstas pueden clasificarse fundamentalmente en cuatro modelos: intracurriculares, intercurriculares, extracurriculares y cursos independientes (Wang, L. 2007); a continuación se define brevemente cada uno de estos modelos.

En relación con el primero, intracurricular, la alfabetización en competencias informacionales se presenta integrada a actividades en un curso o programa, comúnmente mediante una relación de colaboración entre los profesores y el personal de la biblioteca. Los modelos de tipo intercurricular se caracterizan por sesiones adicionales a un curso académico, las cuales son impartidas como respuesta al requerimiento de profesores o responsables de programas. Las propuestas tipo modelo extracurricular suponen que la alfabetización se da por fuera del programa curricular y la participación es voluntaria. Finalmente, en aquellas cuyo tipo de curso es independiente (stand 
alone), la alfabetización informacional tiene lugar en un curso dentro de un currículo académico y está dedicado exclusivamente a este tema; puede tener o no créditos asignados y puede o no ser parte de un programa de la universidad o de una facultad particular. De cada una de estas competencias se derivan formas de actuación que a continuación se describen.

El modelo intracurricular es considerado uno de los más efectivos para el desarrollo de competencias informacionales, lo cual resulta coherente con el planteamiento de Bruce (1997) con respecto a que las acciones en este sentido deben comprometer contenidos específicos de la disciplina, de manera que los estudiantes aprendan aquellos propios de su formación a la vez que desarrollan competencias para buscar y usar información (Bruce, 1997; Feldmann y Feldmann, 2000; Hartmann, 2001). Desde la literatura en cuestión, este argumento ha sido respaldado por varios estudiosos del tema como Cortés (2007); Scaramozzino (2010); Hernández Hernández (2010); Uribe-Tirado y Castaño (2010); Duquesne (2011) y Méndez (2013).

El modelo puede ser gráficamente expresado de la siguiente forma (ver Gráfico 3).

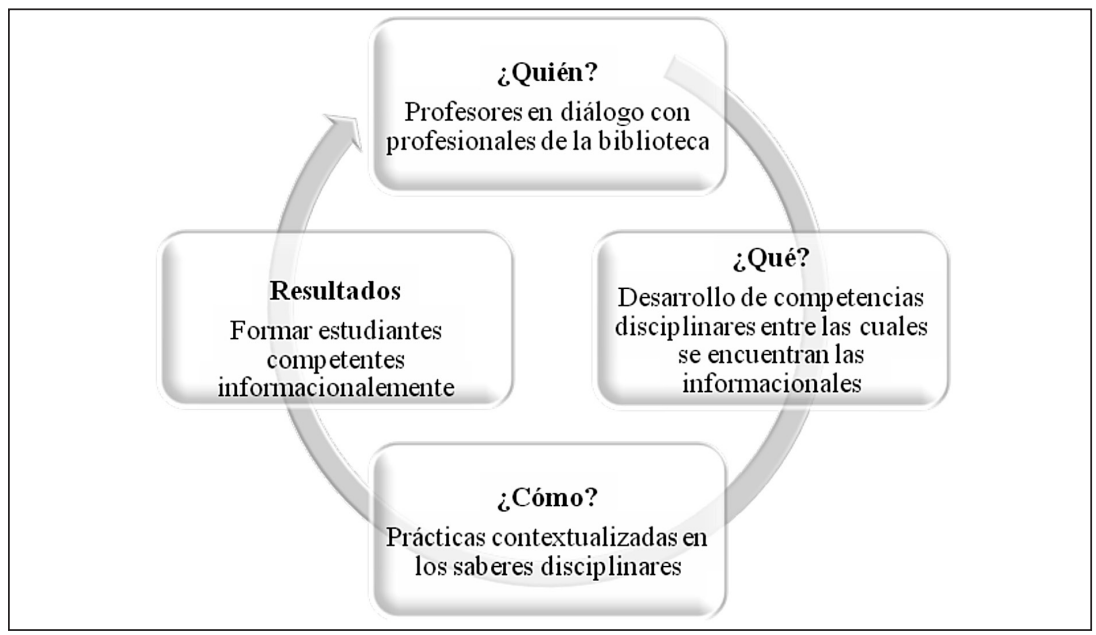

Gráfico 3. Modelo intracurricular.

Fuente: Elaboración propia con base en el texto de L. Wang (2007)

Algo que destacan los autores es que este tipo de modelo revela las posibilidades de rediseñar y negociar las propuestas curriculares a diferentes niveles: institucional, programa académico y cursos, con el fin de garantizar la incorporación de las competencias informacionales coherentemente con los objetivos de formación previstos por los programas académicos. Las pro- 
puestas en este sentido demandan un profundo conocimiento de los contextos en los cuales se va a implementar el modelo así como la constitución de equipos de académicos y bibliotecólogos que trabajen conjuntamente en función de objetivos comunes.

Uno de los estudios en los cuales se refleja este modelo fue adelantado por Tarrant, Dodgson y Law (2008). Otras propuestas en este sentido en general surgen de aproximaciones investigativas en las cuales se hace evidente la necesidad de desarrollar competencias informacionales con acciones claramente incorporadas en los currículos; condición respaldada por los estudios de Wallace, Shorten, y Crookes (2000) y que se reitera en la literatura aquí seleccionada, específicamente en el estudio de Salisbury y Sheridan (2011). Algunos han empleado evaluaciones pretest y postest con el fin de dar cuenta de los cambios experimentados por los estudiantes a partir de diferencias significativas. Los resultados en general sugieren que, si bien algunos estudiantes al inicio del programa se percibían poco competentes, logran mejores resultados al término del programa. No sobra señalar que en estos estudios no se ha contado con instrumentos que evalúen directamente la competencia informacional para enriquecer el reporte verbal de los estudiantes; además, suponer que un cambio en las formas de acceder, evaluar y usar información por parte de los estudiantes pueda ser explicado sin contemplar otros factores quizá resulte algo ingenuo.

Otra de las propuestas que apuntan en este sentido es la de Y. Wang (2007), quien propone la construcción de comunidades de aprendices para el desarrollo de la competencia informacional contando para esto con la intervención de profesionales de la ciencia de la información. La idea es desplazar el foco de atención de prácticas centradas en el profesor o en el profesional de la biblioteca hacia prácticas centradas en el estudiante, en un ambiente colaborativo en el cual es posible que maestros y estudiantes se comprometan en diálogos exploratorios y en actividades para el desarrollo de habilidades y conocimientos que se constituyan en herramientas para reconocer y comprender sus experiencias.

Por su parte, Polkinghorne (2010) expone un enfoque triple para introducir la formación en investigación en un programa de pregrado con énfasis en el desarrollo de competencias informacionales propias del campo disciplinar, en el desarrollo de habilidades en investigación y en la apropiación de métodos de investigación pertinentes para las ciencias políticas. Esta experiencia contó con el trabajo colaborativo entre el personal de la biblioteca y los docentes, lo que hizo posible la adecuación del contenido de los seis talleres implementados a las condiciones propias del campo de formación. Un logro importante fue que los estudiantes desarrollaron mayor conciencia y 
comprensión sobre las habilidades de investigación y su relación con la producción y el análisis de trabajo académico. Se hicieron autoevaluaciones al comienzo y al final así como entrevistas semiestructuradas al terminar el programa; adicionalmente se analizó la producción académica de los estudiantes.

La evaluación del impacto de este tipo de modelos es fundamentalmente cualitativa, del tipo autoreporte, y está centrada en la autopercepción de los estudiantes. Existe un vacío en cuanto al empleo de instrumentos que hagan posible mediciones más precisas sobre cambios en las competencias así como datos sobre los factores asociados a tales cambios. Este es por tanto un campo de investigación inexplorado para lo cual, y como un punto de partida, sería importante tomar como referente los estudios realizados por Meneses-Placeres y Pinto-Molina (2011) en Cuba y el de Marzal García-Quismondo (2010) en España.

El segundo modelo, intercurricular, se caracteriza por la realización de sesiones o módulos adicionales a un curso académico, bien por solicitud del programa o por los docentes. No se encuentran integrados propiamente al currículo sino que se mantienen paralelos, aunque pueden aparecer como parte del programa curricular. Por lo anterior, no demandan un trabajo integrado entre profesores y profesionales de la biblioteca, y cada uno se mantiene dentro de su área de experticia. Un ejemplo de este modelo es presentado en los estudios de Loyola y Matus (2007) en donde la propuesta educativa forma parte de "asignaturas optativas" en los planes de estudio. Gráficamente puede representarse este modelo de la siguiente forma (ver Gráfico 4).

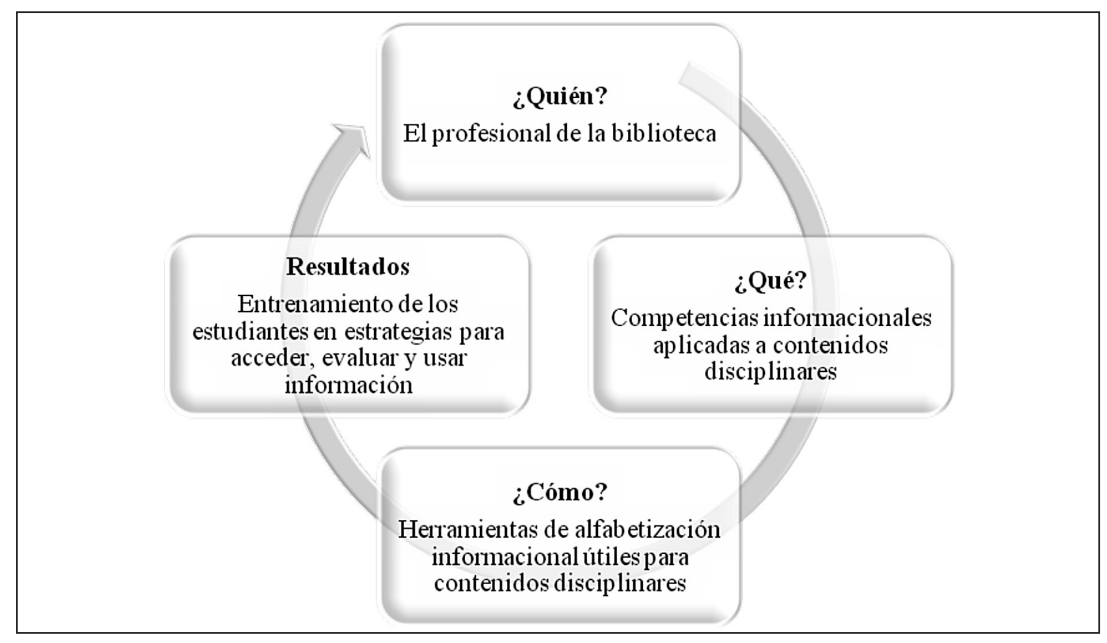

Gráfico 4. Modelo intercurricular 
Ejemplo de este tipo de modelos es el trabajo de Crawford y Broertjes (2010), el cual consistió en desarrollar una unidad obligatoria en línea compuesta por siete módulos y dirigida a introducir a los estudiantes de artes, humanidades y ciencias sociales en habilidades de investigación y búsqueda de información; se usó una evaluación en profundidad de esta experiencia en términos de la percepción que los estudiantes tenían de la misma, información que fue complementada con datos proporcionados por la plataforma de cursos en línea empleada por la institución; en relación específicamente con esto último se tomaron en cuenta los registros sobre las marcas hechas por los estudiantes, los tiempos destinados por ellos a una página, el momento que destinaban a la evaluación y por cuánto tiempo. La información derivada de la evaluación fue empleada para adecuar el programa a las exigencias académicas de los estudiantes y a las necesidades que éstas estimulaban en ellos a lo largo del año.

Por su parte Ku, Sheu y Kuo (2007) adelantaron una investigación con estudiantes de un programa de enfermería que participaron en un curso que contaba con un componente de educación dirigido al desarrollo de competencias informacionales. Los resultados obtenidos con esta experiencia se compararon con un grupo control que participó en otro curso, en el cual no se adelantó acción alguna para el desarrollo de tales competencias; la comparación de resultados dio cuenta de un mejor desempeño en quienes participaron en el grupo experimental. No sobra señalar que un inconveniente de estudios de esta naturaleza es la dificultad para aislar los efectos diferenciados de los cursos por su contenido y metodología, y el aporte de los módulos impartidos para el desarrollo de competencias informacionales.

Una investigación posterior que resulta muy didáctica por la descripción que hace de la experiencia de dos profesores y cursos diferentes es la de Winterman, Donovan y Slough (2011). En este estudio se describen ampliamente los roles desempeñados por el profesor y los bibliotecarios en la planeación del curso así como en su ejecución y evaluación. El uso de portafolios para recoger el proceso y para su evaluación es destacado por su contribución para identificar no solamente un modelo de intervención, sino también estrategias que han sido reconocidas por su utilidad para acompañar experiencias en las que el producto final se construye a lo largo del periodo académico. Si bien la evaluación del modelo se basó únicamente en la confianza percibida en los estudiantes respecto a sus competencias informacionales, permite valorar tales experiencias desde quienes participan en ellas, lo que resulta valioso.

Una vez más se observa la existencia de vacíos en las formas de evaluar el aporte que representa este tipo de modelo para el desarrollo de competencias informacionales. En general los estudios cualitativos retoman los relatos 
de los estudiantes sobre el aprendizaje que creen haber logrado o la confianza ganada a partir de la experiencia respecto a sus habilidades informacionales. Materiales como los portafolios no son objeto de análisis en profundidad así como tampoco los datos que las plataformas institucionales pueden arrojar sobre los itinerarios seguidos por los estudiantes en el proceso. Parecería ser que la riqueza de tales iniciativas no queda documentada por problemas en las formas de evaluarlas.

El modelo extracurricular es quizás el que, dada su naturaleza y condición multicontexto, tiene más cercanía con la "informalidad" en lo formativo. Ejemplos de esta condición son los mostrados en el estudio de Barbosa-Chacón et al. (2010) en donde se evidencia la influencia del contexto laboral sobre el desarrollo de competencias informacionales en estudiantes universitarios que viven esta condición; influencia asociada al cumplimiento de "obligaciones" y "deberes" que conllevan a relaciones con la información; relaciones denominadas por Gómez-Hernández (2007) como "alfabetización informacional organizacional". De igual manera, en el estudio de Barbosa-Chacón et al. (2010) se denota la influencia de los "gustos" personales de los estudiantes frente a sus relaciones con la información; aspecto que pone de manifiesto la "voluntad" para establecer relaciones con la información a partir de intereses y necesidades particulares que pueden estar o no directamente reaccionadas con lo académico. En esta condición los escenarios web resultan ser los más frecuentes para el establecimiento de relaciones con la información, como así lo corroboran los estudios de Loyola y Matus (2007), Hernández Hernández (2010) y Jaramillo, Henniig y Rincón (2011).

El escenario familiar es otro de los contextos que pueden incidir en el desarrollo de la competencia informacional; en ello, la formación y experiencia académica de los integrantes de la familia, especialmente de los padres, puede ser un factor determinante, como lo muestran las investigaciones de Castañeda-Peña et al. (2010) y Cabra et al. (2011) al caracterizar la competencia informacional en nuevos estudiantes universitarios.

En el modelo extracurricular se debe rescatar, también, la incidencia en el desarrollo de la competencia informacional, cuando los estudiantes, no siempre motivados por una responsabilidad académica, se convierten en usuarios de información en interacción con agentes educativos de dichos escenarios; es decir, cuando se establece una labor educativa (Gómez-Hernández, 2010).

Como se aprecia, en este modelo cobra importancia el escenario de desarrollo de la competencia por lo cual la pregunta "en dónde" cobra relevancia. Gráficamente puede representarse este modelo de la siguiente forma (ver Gráfico 5). 


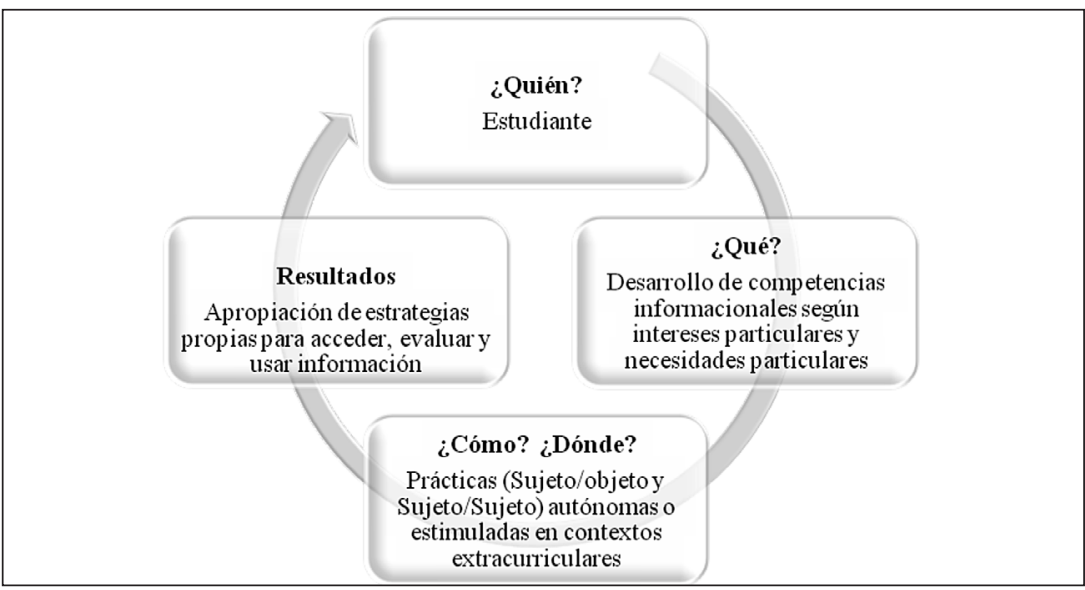

Gráfico 5. Modelo extracurricular

Fuente: Elaboración propia con base en el texto de L. Wang (2007)

En el cuarto modelo tipo curso independiente (stand alone), la alfabetización informacional tiene lugar en un curso específico dentro de un currículo académico. Algo que resulta pertinente precisar es que si bien algunos de tales cursos pueden presentarse como parte del currículo, el tipo de relación que mantienen respecto de las asignaturas que constituyen el plan de estudios es lo que finalmente determina si están integrados o no a la propuesta curricular de un programa de formación profesional. Gráficamente puede representarse este modelo como aparece en el siguiente esquema (ver Gráfico 6).

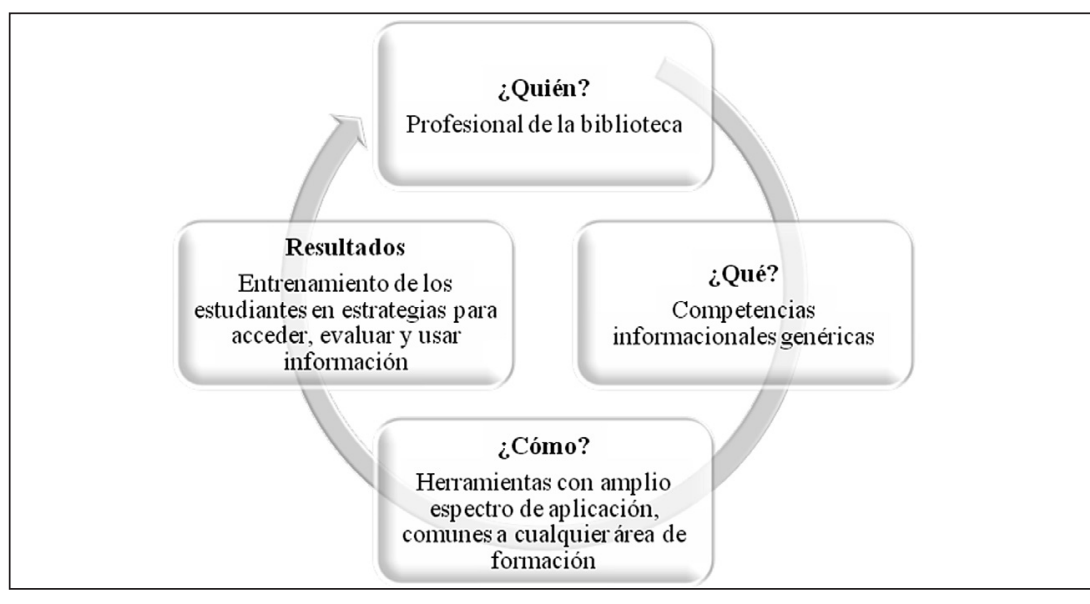


La utilidad de estos cursos ha sido documentada no solamente a nivel de pregrado sino también a nivel doctoral, llegando a realizarse encuestas de carácter nacional encaminadas a aportar evidencias sobre el impacto que pueden tener en la formación de investigadores (Streatfield, Allen y Wilson, 2010). Ilustran este tipo de modelos los resultados de un estudio realizado en Sudáfrica, en el cual participaron 23 universidades y las principales bibliotecas académicas del país. En las universidades se consultó específicamente a los departamentos encargados de los procesos de alfabetización informacional, los que debían informar sobre la forma en que cumplían su cometido en sus respectivas instituciones. De acuerdo con los datos aportados, la mayoría de las acciones dirigidas en las universidades al desarrollo de competencias informacionales se basan en módulos que se imparten dentro del currículo, la mayoría de éstos con créditos asignados y coordinados por los departamentos responsables de alfabetización informacional (Velile Jiyane y Bosire Onyancha, 2010).

Un estudio de menor amplitud es el de Johnston (2010), quien presenta el módulo diseñado para todos los estudiantes de primer año de trabajo social y cuyo propósito fue el desarrollo de habilidades de información y de comunicación. Eligieron realizar un programa en línea debido a las limitaciones del currículo y a las posibilidades de potenciar, fuera del horario académico y con una herramienta de esta naturaleza, el desarrollo de habilidades genéricas para evaluar información, lo que a su vez contribuiría a la formación profesional; los estudiantes debieron completar una evaluación al final para demostrar los resultados de aprendizaje alcanzados. La evaluación del módulo se llevó a cabo mediante grupos focales, observaciones, encuestas y el análisis de las tareas realizadas. Los resultados positivos permitieron a la institución validar la utilidad del módulo así como la importancia del trabajo colaborativo entre docentes y personal de la biblioteca.

Con respecto a la utilidad de este tipo de modelo, Newby (2011) indagó sobre el papel que cumplen los cursos dirigidos a alfabetizar informacionalmente a los estudiantes y concluyó que brindan elementos para acceder a conocimientos disciplinares que no son familiares, así como para conocer la producción académica de autores e instituciones relevantes en su área de interés. Algo que resulta novedoso en una experiencia que realizó fue que, si bien el profesional de la biblioteca no estaba integrado a una propuesta curricular, tampoco estaba solo sino que trabajaba colaborativamente con un investigador de la disciplina que, a su vez, se encontraba participando en una investigación interdisciplinaria; a él le correspondía proporcionar el marco disciplinar donde los problemas del mundo real emergen para integrarlos al aula de clase a través de actividades que pusieran en escena tales proble- 
máticas. Otro aspecto clave de este trabajo fue la manera como se propuso introducir a los estudiantes en culturas disciplinares que les eran ajenas, de manera que se fueran apropiando de fenómenos, conceptos, teorías y aspectos epistemológicos fundamentales para comprender la estructura de tales campos de conocimiento. Con esta propuesta se buscó formar estudiantes de pregrado que aprendieran a trabajar interdisciplinariamente.

Un estudio que aporta elementos valiosos porque logra concentrar tres experiencias con tres modelos diferentes (intracurricular, intercurricular e independiente) es el de Detlor et al. (2011). En una escuela de negocios analizan los impactos de programas basados en modelos diferentes para el desarrollo de competencias informacionales. Los resultados, valorados a partir del criterio de los bibliotecólogos, profesores y estudiantes de pregrado involucrados, son mejores cuando se emplean modelos intracurriculares e intercurriculares, particularmente con el primer modelo; logros menos importantes se reportan a partir de experiencias tipo curso independiente. Igualmente resulta relevante el estudio sobre la utilidad del modelo intracurricular en la medida en que supera la falta de participación de los estudiantes por ser cursos voluntarios, así como la incorporación de talleres en los cuales los aprendizajes son aplicados a contenidos propios del campo de formación profesional de los participantes. Los programas basados en modelos intracurriculares hacen posible conceder a los estudiantes un tiempo mayor para la asimilación de contenidos así como para el desarrollo de competencias y favorecen la continuidad entre cada clase. Estos beneficios respecto a los otros modelos explican, en parte, los resultados superiores en términos de competencias informacionales obtenidos por los estudiantes que participaron en tales experiencias al compararlos con los demás estudiantes.

Un quinto modelo que emerge en esta revisión, y que no fue anunciado previamente, es el que llevan a cabo los programas de pregrado en Ciencias de la Información. El modelo podría denominarse simplemente como curricular, en tanto que se atribuye a la propuesta del plan de estudios y demás actividades de la carrera el desarrollo de competencias informacionales sin acudir a acciones adicionales, bajo el supuesto de que la comunidad disciplinar introducirá a los nuevos profesionales de la ciencia de la información en las prácticas propias de la cultura disciplinar. El modelo podría representarse como aparece en el Gráfico 7. 


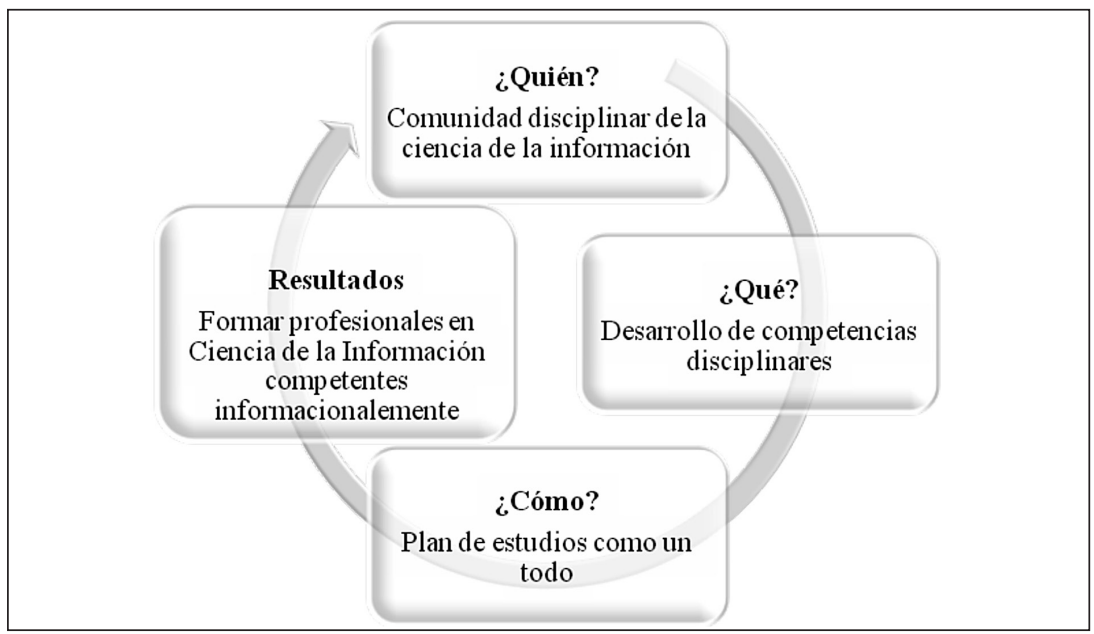

Gráfico 7. Modelo Curricular

Fuente: Elaboración propia

Este modelo se evidencia en el estudio de Islam y Tsuji (2010) en el cual se buscó evaluar el desarrollo de competencias informacionales en los egresados de Ciencia de la Información mediante cuestionarios y entrevistas. Los resultados poco satisfactorios son explicados por los investigadores con la ausencia de un curso específico en el currículo dirigido a su desarrollo. Este resultado es relevante en tanto que evidencia la insuficiencia de las prácticas culturales de la disciplina, actualizadas en los espacios de aprendizaje propios de la formación en esta área para desarrollar este tipo de competencias. Un aporte adicional de este estudio es la reflexión que se hace sobre la necesidad de contar con políticas nacionales en este sentido, que guíen las acciones en esta línea, las cuales han de iniciar en la escuela.

Como pudo apreciarse, cada uno de los modelos presentados ofrece posibilidades para implementar propuestas curriculares que involucren a la institución y al programa académico para el desarrollo de competencias informacionales que tomen en cuenta los objetivos de formación, con márgenes de negociación entre los profesionales de ciencia de la información y los docentes, variables que dependen de la integración, o no, de las propuestas en los programas de formación. Con independencia del modelo que se adopte, algo que reiteradamente se destaca en las investigaciones y experiencias es el valor del trabajo colaborativo entre los profesionales de la biblioteca y los docentes para fortalecer las competencias de los estudiantes (Marciales et al., 2013). 


\section{Estrategias para el Desarrollo de Competencias Informacionales}

Se entienden las estrategias como el conjunto de acciones y herramientas a través de las cuales se busca generar cambios en las competencias para acceder, evaluar y hacer uso de información por parte de los estudiantes; tales estrategias no toman en cuenta los currículos para su implementación.

Algunos artículos revisados se focalizan fundamentalmente en la identificación de estrategias de enseñanza/aprendizaje para estimular el desarrollo de competencias informacionales usando programas en línea, o empleando motores de búsqueda y bibliotecas digitales, el uso del correo electrónico y los weblogs, entre otros. Estas estrategias son promovidas en la academia mediante espacios en los cuales se anima a los estudiantes a emplearlas en contextos diferentes al escenario formal académico (Cortés, 2007; Loyola y Matus, 2007; Valadez et al., 2007; Hanson y Williams, 2008; Cabral, 2008; Rincón, 2009; Bowler, 2010; Hernández Hernández, 2010; Uribe y Castaño, 2010; Rastgoo et al., 2011; Pineda et al., 2012; Gómez-Hernández, 2013). Con tales herramientas y con las tareas académicas como instrumentos psicológicos se busca incidir sobre procesos metacognitivos. Éstos son potenciados a medida que el aprendiz valora fuentes de información y define criterios para hacer tal valoración (Pineda et al., 2012); condición que es favorecida si se cuenta con bibliotecarios en el rol particular de "formadores" o, en especial, como "mentores" (Pinto y Uribe, 2010). Se desprende de tales estudios que el desarrollo cognitivo alcanzado por los estudiantes a través de las tareas académicas estimula la confianza en sí mismos como aprendices y los prepara para el aprendizaje a lo largo de toda la vida.

El uso de proyectos para involucrar a los estudiantes en procesos de solución de problemas propios de su disciplina es otra estrategia que ha sido utilizada (Cortés, 2007; Duquesne, 2011; Yua et al., 2011; Álvarez, Ríos y Velásquez, 2012). Algo que destacan los autores es el hecho de que la instrucción específica para el desarrollo de competencias informacionales no es requerida en este tipo de experiencias, pues las exigencias de la tarea es lo que contribuye fundamentalmente a su desarrollo.

Otros de los estudios sobre estrategias para el desarrollo de competencias informacionales son los de Loyola y Matus (2007) y McClure, Cooke y Carlin (2011); estudios relacionados con programas que analizan estrategias en línea para la instrucción en competencias escriturales que demandan el uso de fuentes de información. Resulta interesante en estos estudios la integración que se hace entre procesos escriturales y competencias informacionales, a través de una tarea como la escritura de un ensayo, ante lo que el estudiante se ve exigido a seleccionar recursos impresos y digitales para acceder a fuentes de 
información, construir criterios para seleccionarlas, citarlas, tomar en cuenta la actualidad, autoridad y calidad de las mismas, y elaborar un texto con sentido que siga las reglas gramaticales de manera coherente con el género textual elegido. En situaciones análogas a las de estos estudios, son rescatables los estudios de Cortés (2007) en México y Pineda et al. (2012) en Colombia, en los que se destaca, como estrategia, la realización de ejercicios conducentes a que los educandos valoren y respeten la producción intelectual.

En el estudio de Niedbala y Fogleman (2010) se emplea la comunicación ubicua para desarrollar estas competencias utilizando como estrategia la enseñanza sobre el uso de la biblioteca, el andamiaje del proceso de aprendizaje para mejorar la comprensión de grandes cantidades de información en la investigación en línea y la evaluación entre pares dentro de una clase. La tecnología ubicua permite a los individuos aprender allí donde estén, y contar para ello con los componentes de su entorno social. En esta investigación se emplearon wikis para mejorar las prácticas de investigación de los estudiantes así como sus resultados académicos; asimismo, herramientas de la web 2.0 se integraron al aula de clase para facilitar el aprendizaje colaborativo. Esta combinación de estrategias condujo a resultados positivos que merecen ser revisados por quienes trabajan con estas herramientas.

El estudio con profesores en formación se centró fundamentalmente en el aporte que la evaluación formativa dada por los bibliotecólogos representa para el mejoramiento de las competencias informacionales (Robertson et al., 2011). Se indagó inicialmente sobre la habilidad de los profesores para evaluar críticamente fuentes de información; competencia que algunos sugieren que es sobreestimada por los profesores en sí mismos (Wang, Y. 2007; Cortés, 2007; Duquesne, 2011). La evaluación formativa se entendió como aquella retroalimentación que se da al estudiante antes de entregar el documento final de un proyecto y está dirigida a darle la oportunidad de revisar su aprendizaje (Johnson y Jenkins, 2009). El estudio demostró el aporte de este tipo de evaluaciones para apoyar el aprendizaje de los estudiantes y para mejorar las prácticas de enseñanza.

Estudios en donde se destaca la importancia de contar con docentes preparados y que propongan la integración de estas competencias son los de Méndez (2008) en Chile y los de Pineda et al. (2012) y Méndez (2013) en Colombia; allí se demuestra que contar con esta condición y la responsabilidad que representa es factor crítico para el éxito de los alumnos en su aprendizaje.

Algunas alternativas que rompen con las formas más tradicionales de desarrollar la competencia informacional son recogidas en el artículo de Smale (2011), en el cual se describe el uso de juegos no solamente digitales, a través de los cuales se busca potenciar el aprendizaje y también la motivación me- 
diante estrategias que pueden proporcionar estructura y organización para una tarea compleja, como es la de acceder, evaluar y usar información. Estas propuestas se sustentan en los aportes de estudios de corte cognitivista, en los cuales se destacan los beneficios del aprender haciendo (Loyola y Matus, 2007; Cortés, 2007; Valadez et al., 2007; Robertson et al., 2011). Experiencias similares en las cuales se emplearon juegos digitales se relatan en Allgood y Gallegos (2007) y Markey et al. (2009); algunos emplean incluso herramientas no digitales (Smith, 2007). Se recogen en este último artículo una serie de estudios en los cuales se describe brevemente la orientación de los juegos según los aspectos de competencia informacional que se pretenden trabajar.

Este tipo de estrategias se proponen como instrumentos pertinentes cuando existe poco contacto entre los bibliotecólogos y los estudiantes, lo que hace difícil captar la atención y motivación de estos últimos. No obstante los aportes que ofrecen herramientas de este orden, existen limitaciones para su empleo como los costos y tiempos que demanda su desarrollo, y la necesidad de contar con un equipo de profesionales que lleve a cabo el mantenimiento y actualizaciones que se requieren para evitar su desactualización. Como enseñanza de estos estudios se deriva la no necesidad de llevar a cabo juegos complicados para el desarrollo de competencias informacionales y la gran utilidad que tienen los juegos simples con objetivos claros y evaluables.

Otros aportes en este sentido son los de Regneală, quien sintetiza las acciones que en Rumania se vienen haciendo para lograr contar con una población alfabetizada informacionalmente, lo que ellos denominan como formar una cultura informatiei (2010: 130), propuesta definida por consenso en 2009 en la XX-th National Conference of the Romanian Library Association (ABR), con la participación de los bibliotecólogos rumanos; en este evento se creó también la sección de alfabetización informacional de la asociación. Lo que resulta valioso de este trabajo es la presentación que hace de manera sucinta de los elementos estructurales y estratégicos de una iniciativa de alfabetización informacional que impacta los ámbitos locales y nacionales, y que toma como referentes las acciones que en el contexto internacional más amplio se están llevando a cabo para formar a la población de cara a los retos del siglo XXI. Además, un valioso aporte de esta experiencia es el hecho de que emerge de un consenso entre profesionales de la información que piensan un problema común e integran esfuerzos hacia propósitos compartidos. Lo que se constituye como marco orientador de toda acción que se emprenda en contextos educativos e inclusive en espacios laborales que demandan este tipo de habilidades.

Los proyectos en los que se visualiza una amplia gama de estrategias adicionales son los estudios de Loyola y Matus (2007) en Chile; Cortés (2007) y 
Valadez et al. (2007) en México; Uribe-Tirado y Castaño (2010) y Jaramillo, Henniig y Rincón (2011) en Colombia; estudios en donde, en materia de desarrollo de cursos formales, cabe destacar: i) diagnósticos de "entrada" como base de intervención; ii) metodología cualitativa para rescatar la percepción de los alumnos en torno a su aprendizaje y habilitación de las nuevas competencias; iii) desarrollo de procesos de formación por etapas en función de la cultura organizacional; iv) redes de cooperación entre académicos para abordar la tarea de alfabetización en información universitaria; v) utilización de contenidos sobre información con base en diagnósticos por asignaturas; vi) "aprender a aprender" aplicando un juicio crítico ante la información; vii) transferir lo aprendido a otros contextos (familiares, amigos, niveles educativos precedentes); viii) formulación de lineamientos para el fomento de las competencias informacionales a nivel institucional y ix) articulación de las asignaturas ALFIN con los modelos educativos en donde tienen presencia.

Acciones igualmente ambiciosas son aquellas que Katz (2007) presenta, en las cuales se busca agenciar un proyecto de orden nacional para la alfabetización informacional de la población. En agosto de 2003 se estableció el National Higher Education ICT Literacy Initiative, consorcio integrado por siete colleges y universidades que reconocían la necesidad de integrar la alfabetización informacional a la educación secundaria. Representantes de las diferentes instituciones colaboraron en el diseño y desarrollo de un sistema de evaluación de tales competencias. De ese trabajo se derivó una evaluación basada en la evidencia dirigida a identificar las habilidades de los estudiantes para emplearla como información de base para el desarrollo de programas de alfabetización informacional. Los datos obtenidos llevaron a la creación del National ICT Literacy Policy Council, encargado de delinear los estándares en alfabetización informacional de manera que sirvieran como orientación sobre lo que los estudiantes deberían lograr y cómo hacerlo en los diferentes niveles académicos.

En lo relativo al desarrollo de tutoriales vale la pena retomar el trabajo de Aydelott (2007) basado en los estándares de la Association of College and Resesarch Libraries (ACRL) (2000) para estudiantes de pregrado de ciencias e ingeniería así como para aquellos ya graduados. Un primer paso de este proyecto consistió en identificar cuáles serían las competencias cuyo desarrollo era responsabilidad de los bibliotecarios a fin de no traspasar los límites de la disciplina e invadir terrenos propios de la formación profesional. El tutorial incluye vínculos a contenidos disciplinares específicos y algunas evaluaciones para el estudiante, así como listas con herramientas, técnicas y recursos didácticos de la biblioteca, de manera que este instrumento se configure como un centro de autoayuda para acceder a fuentes de documentación útiles para 
el estudiante. Un propósito importante de esta herramienta que es destacado por el autor es que no se pretende lograr un punto final en una propuesta de alfabetización informacional como ésta, sino sentar las bases para el desarrollo de aquellas habilidades requeridas en el contexto de la sociedad de la información que además son desarrolladas y perfeccionadas a lo largo del tiempo y durante toda la vida.

\section{Conclusiones}

En los últimos cinco años dos enfoques han orientado la investigación y la intervención sobre competencias informacionales: el enfoque cognitivo y el sociocultural. El primero ha aportado herramientas valiosas para la comprensión de factores de orden cognitivo y metacognitivo que intervienen en la forma como los sujetos acceden, evalúan y usan la información. El valor de estos trabajos ha sido precisamente el de proporcionar herramientas teóricas para orientar procesos. La introducción del enfoque sociocultural resalta el carácter situado de las competencias y su relación con factores de orden social y cultural.

En relación con los modelos, si bien los métodos de evaluación registran falencias, las aproximaciones que se han hecho permiten documentar algunos de los beneficios obtenidos en función de los propósitos formativos perseguidos. El estudio que brinda elementos más significativos en cuanto al aporte que reviste cada tipo de modelo es el de Detlor et al. (2011). De acuerdo con éste, los mejores resultados se obtienen empleando modelos intracurriculares, posiblemente porque la continuidad entre las clases favorece la asimilación de contenidos.

No obstante lo anterior, la decisión de implementar uno u otro de los modelos documentados es compleja en tanto supone la movilización de recursos de diverso orden, así como la adecuación de los tiempos académicos y de los programas curriculares a exigencias que no han ocupado un lugar legítimo especialmente en la formación universitaria.

En relación con las estrategias, si bien parecería que la introducción de herramientas de la Web 2.0 contribuyen al desarrollo de competencias, lo que habría que destacar son los recursos utilizados como el uso de la biblioteca, la participación de los maestros en el andamiaje del proceso para mejorar la comprensión de grandes cantidades de información, así como la introducción de la evaluación entre pares. Más que el uso de wikis habría que destacar la pregunta implícita que existe en las experiencias señaladas, la cual indaga sobre cómo mejorar las prácticas de investigación de los estudiantes sin res- 
tringir el desarrollo de competencias informacionales a aquellas propias de la actividad investigativa. Respecto al papel de los maestros cabe destacar el uso de la evaluación formativa entendida como la retroalimentación que contribuye a orientar el trabajo de los estudiantes antes de obtener una nota. No resulta sorprendente encontrar que este tipo de evaluaciones apoya el aprendizaje y contribuye a mejorar las prácticas de enseñanza.

Por otra parte, la introducción de experiencias lúdicas para el desarrollo de competencias informacionales parecería tener valor para captar la atención y motivación de los estudiantes. Su uso debe tener en cuenta no solamente su costo y el tiempo que demandará su desarrollo sino también la necesidad de actualización, lo cual solamente es posible si existe un equipo de colaboradores encargados de esto, y no solamente iniciativas loables, pero aisladas, de maestros y bibliotecólogos preocupados por el aprendizaje.

Para terminar solamente resta señalar la existencia de áreas inexploradas de investigación así como la poca preocupación demostrada por precisar los referentes epistemológicos y teóricos que sustentan los modelos y estrategias adoptados. Posiblemente una reflexión más profunda en este sentido, antes de emprender múltiples acciones, contribuiría a hacer desplazamientos en la intervención que tomen distancia de enfoques asumidos más por tradición que por reflexión, y que comiencen a incluir la cultura como pregunta para jalonar desde allí procesos de desarrollo de la competencia informacional, contextuados y situados.

\section{REFERENCIAS}

Allgood, T. y Gallegos, B. (2007), Quarantined: Axl Wise and the Information Outbreak: Creating an online game to teach information literacy skills. Disponible en: http://gaming.techsource.ala.org/ images/e/ec/Quarantined_techsource.pdf [Fecha de consulta: 3/07/2012].

Álvarez, J. C.; Ríos, I. y Velásquez, E. (2012), “CCómo desarrollar habilidades para la obtención de información científica? Una propuesta desde la clase de Física", en Revista IPLAC, núm. 1. Disponible en: http://www.revista.iplac.rimed.cu/index.php?option=com _content\&view $=$ article \&id=588:icomo-desarrollar-habilidades-pa ra-la-obtencion-de-informacion-cientifica-una-propuesta-desde-laclase-de-fisica\&catid=53:12-1-e\&Itemid=265 [Fecha de consulta: 30/12/2013].

ACRL (Association of College and Research Libraries) (2000), Information Literacy Competency Standards for Higher Education. Disponible en: http://www.ala.org/ala/acrl/acrlstandards/information literacycompetency.html [Fecha de consulta: 30/10/2009]. 
Aydelott, K. (2007), "Using the ACRL Information Literacy Competency Standards for Science and Engineering/Technology to Develop a Modular Critical-Thinking-Based Information Literacy Tutorial”, en Science E Technology Libraries, vol. 27, núm. 4, pp. 19-42.

Barbosa-Chacón, J.; Barbosa, J.; Marciales, G. y Castañeda-Peña, H. (2010), "Reconceptualización sobre las competencias informacionales. Una experiencia en la Educación Superior", en Revista de Estudios Sociales, vol. 37, pp. 121-142.

Bowler, L., (2010), "Taxonomy of adolescent metacognitive knowledge during the information search process", en Library \& Information Science Research, vol. 32, núm. 1, pp. 27-42.

Bruce, C. S. (1997), The seven faces of information literacy, Adelaide: Auslib Press.

Cabral, B. (2008), "La biblioteca digital y la educación a distancia como entes inseparables para incrementar la calidad de la educación”, en Investigación Bibliotecológica, vol. 22, núm. 45, pp. 63-78. Disponible en: http://cuib.unam.mx/revistaCuib.html [Fecha de consulta: 30/12/2013].

Cabra, F.; Marciales, G.; Gualteros, N. y Mancipe, E. (2011), “Dimensiones socioculturales de la competencia informacional en estudiantes universitarios: creencias, cultura y experiencias vitales", en Revista Iberoamericana de Educación, vol. 26, núm. 4, pp. 1-12.

Castañeda-Peña, H.; González, L.; Marciales, G.; Barbosa-Chacón, J. y Barbosa, J. (2010), "Recolectores, verificadores y reflexivos: perfiles de la competencia informacional en estudiantes universitarios de primer semestre", en Revista Interamericana de Bibliotecología, vol. 33, núm. 1, pp. 187-209.

Cope, B. y Kalantzis, M. (2009), "Multiliteracies: New Literacies, New Learning. Pedagogies”, en An International Journal, vol. 4, núm. 3, pp. 164-195.

Cortés, J. (2007), “El desarrollo de competencias informativas en estudiantes universitarios a través de un curso con valor en créditos", en Biblios, vol. 29, pp. 1-12.

Crawford, N. y Broertjes, A. (2010), "Evaluation of a university online. Information Literacy unit", en Australian Library Journal, vol. 59, núm. 4, pp. 187-196.

Detlor, B.; Julien, H.; Willson, R.; Serenko, A. y Lavallee, M. (2011), "Learning Outcomes of Information Literacy Instruction at Business Schools", en Journal of the American Society for Information Science and Technology, vol. 62, núm., 3, pp. 572-585.

Dewey, J. (1938). Experience and Education, New York: Simon and Schuster.

Duquesne, A. (2011), "La alfabetización en información en los Policlínicos Universitarios”, en Educación Médica Superior, vol. 25, núm. 2, pp. 157-163.

Eisenberg, M.; Lowe, C. y Spitzer, K. (2004), Information Literacy: Essential Skills for the Information Age, London: Libraries Unlimited. 
Feldmann, L. y Feldmann, J. (2000), Developing information literacy skills in freshmen engineering technology students, artículo presentado en la 30th Annual Frontiers in Education Conference (Kansas City, EUA, 18-21 octubre de 2000).

Gómez-Hernández, J. (2007), Alfabetización Informacional; cuestiones básicas, Grupo Thinkepi. Estrategia y Prospectiva de la información. Disponible en: http://www.thinkepi.net/alfabetizacion-infor macional-cuestiones-basicas [Fecha de consulta: 22/01/2014]. - (2010), "Las bibliotecas universitarias y el desarrollo de las competencias informacionales en los profesores y estudiantes", en Revista de Universidad y Sociedad del Conocimiento, vol. 7, núm. 2, pp. 39-49. - (2013), "Unir Educación y Bibliotecas: La evolución de un reto permanente”, en El Profesional de la Información, vol. 22, núm. 2, pp. 101-105.

Gutiérrez, A. y Tyner, K. (2012), "Educación para los medios, alfabetización mediática y competencia digital”, en Revista Científica de Educomunicación, vol. 38, núm. XIX, pp. 31-39.

Hanson, J. y Williams, J. M. (2008), "Using writing assignments to improve self-assessment and communication skills in an engineering statics course", en Journal of Engineering Education, vol. 4, pp. 515-529.

Hanson, R. (1958), Observation and explanation: A guide to philosophy of science. Patterns of discovery and inquiry into the conceptual foundation of science, Cambridge: University Press.

Hartmann, E. (2001), "Understandings of information literacy: The perceptions of first year undergraduate students at the University of Ballarat", en Australian Academic E Research Libraries, vol. 32, núm. 2, pp. 110-122.

Hernández Hernández, C. (2010), "Un plan de formación en competencias de información a través de aulas virtuales: análisis de una experiencia con alumnado universitario”, en Revista de Universidady Sociedad del Conocimiento, vol. 7, núm. 2, pp. 50-62.

Islam, M. y Tsuji, K. (2010), "Assessing information literacy competency of Information Science and Library Management graduate students of Dhaka University Mohammed Anwarul Islam University of Dhaka", en International Federation of Library Associations and Institutions, vol. 36, núm. 4, pp. 300-316.

Jaramillo, P.; Henniig, C. y Rincón, Y. (2011), “¿Cómo manejan información los estudiantes de educación superior? El caso de la Universidad de La Sabana, Colombia”, en Información, Cultura y Sociedad, vol. 25, pp. 117-143.

Jenkins, H.; Clinton, K.; Robison, A.; Weigel, M. y Purushotma, R. (2006), Confronting the Challenges of Participatory Culture: Media Education for the 21st Century. Disponible en: http://digitallearning.macfound.org/atf/cf/\%7B7E45C7E0-A3E0-4B89-AC9C-E807 E1B0AE4E\%7D/JENKINS_WHITE_PAPER.PDF [Fecha de consulta: 15/11/2012]. 
Johnson, E. y Jenkins, J. (2009), "Formative and summative assessment”, en Eric M. Anderman y Lynley H. Anderman, Psychology of classroom learning: An encyclopedia, Detroit: Macmillan.

Johnston, N. (2010), "Is an online learning module an effective way to develop information literacy skills?", en Australian Academic \& Research Libraries, vol. 41, núm. 3, pp. 207-218.

Katz, I. (2007), "Testing Information Literacy in Digital Environments: ETS's iSkills Assessment”, en Information Technology and Libraries, vol. 26, núm. 3, septiembre, pp. 3-12.

Kozulin, A. (2000), Instrumentos psicológicos. La educación desde una perspectiva sociocultural, Barcelona: Paidós.

Ku, Y.; Sheu, S. y Kuo, S. (2007), "Efficacy of Integrating Information Literacy Education Into a Women's Health Course on Information Literacy for RN-BSN Students", en Journal of Nursing Research, vol. 15, núm. 1, pp. 67-77.

Loyola, E. y Matus, G. (2007), "La alfabetización en Información: un primer paso en la formación de los Bibliotecólogos en la Universidad de Playa Ancha”, en Revista Faro, vol. 5. Disponible en http:// alfiniberoamerica.wikispaces.com/Chile [Fecha de consulta: 15/11 /2012 ]

Marciales Vivas, G. P.; González, L.; Castañeda-Peña, H. y Barbosa-Chacón, J. (2008), "Competencias Informacionales en Estudiantes Universitarios: una reconceptualización”, en Universitas Psychologica, vol. 7, núm. 3, pp. 643-654.

_ ; Cabra, F.; Castañeda-Peña, H.; Peña, L.; Mancipe, E. y Gualteros, N. (2013), Nativos digitales: transiciones de lo impreso a lo digital (en prensa).

__ ; Barbosa, J. C.; Rico, C. y Rubiano, C. (2006), "Aprendizaje y Sociedad de la Información”, en Saber, Sujeto y Sociedad: Una Década de Investigación en Psicología, Bogotá: Editorial Pontificia Universidad Javeriana. (Colección Biblioteca del Profesional.)

Markey, K.; Swanson, F.; Jenkins, A.; Jennings, B.; St. Jean, B.; Rosenberg, V. y Frost, R. L. (2009), "Will undergraduate students play games to learn how to conduct library research?", en Journal of Academic Librarianship, vol. 35, núm. 4, pp. 303-313. Disponible en: http://www.elsevier.com/locate/jacalib [Fecha de consulta: 3/07/2012].

Marzal García Quismondo, M. A. (2010), “La evaluación de los programas de alfabetización en información en la educación superior: estrategias e instrumentos", en Revista de Universidad y Sociedad del Conocimiento, vol. 7, núm. 2, pp. 28-38.

McClure, R.; Cooke, R. y Carlin, A. (2011), "The Search for the Skunk Ape: Studying the Impact of an Online Information Literacy Tutorial on Student Writing”, en Journal of Information Literacy, vol. 5, núm. 2, pp. 26-45. Disponible en: http://ojs.lboro.ac.uk/ ojs/index.php/JIL/article/view/PRA-V5-I2-2011-2 [Fecha de consulta: 26/12/2012]. 
Méndez, J. C. (2013), "La alfabetización informacional en la educación superior: una mirada desde el análisis crítico del discurso", en Uni-pluri/versidad, vol. 13, núm. 2, pp. 60-78.

Méndez, M. (2008), "Propuesta de competencia en gestión de información y niveles de desempeño para la docencia universitaria”, en Revista Electrónica de Desarrollo de Competencias, vol. 2, núm 2, pp. 16-38.

Meneses-Placeres, G. y Pinto-Molina, M. (2011), “Alfinev. Modelo para evaluar la alfabetización informacional en la educación superior en Cuba", en Investigación Bibliotecológica, vol. 25, núm. 55, pp. 81-106. Disponible en: http://www.scielo.org.mx/scielo.php? script=sci_arttext $\&$ pid $=$ S0187-358X2011000300004 [Fecha de consulta: 30/11/2013].

Milne, C. (2004), “University of Abertay, Dundee”, en SCONUL (ed.), Learning Outcomes and Information Literacy, London: SCONUL, pp. 9-21.

Montiel-Overall, P. (2007), "Information Literacy: Toward a Cultural Model", en Canadian Journal of Information and Library Science (Special Edition on Information Literacy), vol. 31, núm. 1, pp. 43-68.

Newby, J. (2011), "Entering unfamiliar territory building an information literacy course for graduate students in interdisciplinary areas", en Reference E User Services Quarterly, vol. 50, núm. 3, pp. 224229.

Niedbala, M. y Fogleman, J. (2010), "Taking Library 2.0 to the Next Level: Using a Course Wiki for Teaching Information Literacy to Honors Students", en Journal of Library Administration, vol. 50, pp. 867-882.

Pineda, C.; Hennig, C.; Segovia, Y. y Díaz, D. (2012), "Alfabetización informacional en la educación superior virtual: logros y desafíos”, en Información, Cultura y Sociedad, vol. 26, pp. 83-104.

Pinto, M. y Uribe, A. (2010), "Formarnos y autoformarnos en alfabetización informacional. Un programa de mentorización en bibliotecas universitarias-CRAI", en Investigación Bibliotecológica, vol. 24, núm. 52, pp. 63-95. Disponible en: http://cuib.unam.mx/revis taCuib.html [Fecha de consulta: 25/12/2013].

Polkinghorne, S. (2010), "Research is a verb: Exploring a new information literacy-embedded undergraduate research methods course", en Canadian Journal of Information and Library Science, vol. 34, núm. 4, pp. 457-473.

Rastgoo, A.; Naderi, E.; Shariatmadari, A. y Seifnaraghi, M. (2011), "Investigating the Effect of Internet Information Literacy on the Development of University Students' Metacognitive Skills”, en Australian Journal of Basic and Applied Sciences, vol. 5, núm. 8, pp. 959-968.

Regneală, M. (2010), "Information Literacy Instruction-The Major Challenge of Contemporary Library Science”, en Library and Information Science Research, vol. 14, pp. 130-136. 
Rincón Gallardo, J. T. (2009), The effect of the use of learning journals on the development of metacognition in undergraduate students. Doc. inéd. Ph. D Dissertation, Capella University.

Robison, A. (2010), “New Media Literacies by Design”, en K. Tyner (ed.), Media Literacy. New Agendas in Communication, New York \& London: Routledge.

Robertson Seely, S.; Winstead Fry, S. y Ruppel, M. (2011), "Information Literacy Follow-Through: Enhancing Preservice Teachers' Information Evaluation Skills Through Formative Assessment", en Behavioral E Social Sciences Librarian, vol. 30, pp. 72-84.

Salisbury, F. y Sheridan, L. (2011), "Mapping the journey: Developing an information literacy strategy as part of curriculum reform", en Journal of Librarianship and Information Science, vol. 43, núm. 3, pp. 185-193.

Scaramozzino, J. (2010), "Integrating STEM Information Competencies into an Undergraduate Curriculum", en Journal of Library Administration, vol. 50, núm. 4, pp. 315-333.

Shaffer, D. W.; Squire, K. R.; Halverson, R. y Gee, J. P. (2005), "Video games and the future of learning", en Phi Delta Kappan, vol. 87, núm 2, pp. 105-111. Disponible en: http://www.kappanmagazine. org/ [Fecha de consulta:3/07/2012].

Smale, M. A. (2011), "Learning Through Quests and Contests: Games in Information Literacy Instruction", en Library Innovation, vol. 2, núm. 2, pp. 36-55.

Smith, F. A. (2007), "Games for teaching information literacy skills", en Library Philosopby \& Practice, vol. 9, núm. 2, pp. 1-12. Disponible en: http://www.webpages.uidaho.edu/ mbolin/lpp.htm [Fecha de consulta: 4/08/2012].

Streatfield, D.; Allen, D. y Wilson, T. (2010), "Information Literacy Training for Postgraduate and Postdoctoral Researchers: a National Survey and its Implications", en International Journal of Libraries and Information Services, vol. 60, núm. 3, pp. 230-240.

Tarrant, M.; Dodgson J. E. y Law, B. (2008), “An approach to curriculum for improving the Information literacy Skills and academic Writing of Students in the Field of nursery in Hong Kong”, en Nurse Education Today, vol. 28, pp. 458-468.

Uribe-Tirado, A. (2009), Interrelaciones entre veinte definiciones-descripciones del concepto de alfabetización informacional: propuesta de macro-definición. Disponible en: http://eprints.rclis.org/bits tream/10760/14145/1/20_definiciones_ALFIN.zip [Fecha de consulta: $14 / 01 / 2013]$.

y Castaño, W. (2010), "La formación en competencias informáticas e informacionales desde la Escuela Interamericana de Bibliotecología con el apoyo de una plataforma de e-learning. Experiencias y resultados", en Revista Venezolana de la Información, vol. 2, núm. 2. Disponible en: http://eprints.rclis.org/17875/1/uri be_y_castano_mp_v2n2.pdf [Fecha de consulta: 30/12/2013]. 
Valadez, R.; Páez, J.; Zapata, M.; Espinosa, J.; Cortés, X. y Monroy, M. (2007), "Diseño de un curso en línea para la alfabetización informacional bajo el modelo ADDIE: una experiencia en la UNAM", en Revista de Sistemas de Información y Documentación, vol. 1, pp. 267-274.

Velile Jiyane, G. y Bosire Onyancha, O. (2010), “Information literacy education and instruction in academic libraries and LIS schools in institutions of higher education in South Africa”, en South African Journal of Libraries EI Information Science, vol. 76, núm. I, pp. 11-23.

Verhey, M. P. (1999), "Information literacy in an undergraduate nursing curriculum: development, implementation, and evaluation", en Journal of Nursing Education, vol. 38, núm. 6, pp. 252-259.

Wallace, M.; Shorten, A. y Crookes, P. (2000), “Teaching information literacy skills: an evaluation”, en Nurse Education Today, vol. 20, núm. 6, pp. 485-489.

Wang, L. (2006), "Information literacy courses: a shift from a teacher-centred to a collaborative learning environment", en 4th International Lifelong Learning Conference: Partners, Pathways, and Pedagogies (Yeppoon, Queensland, Australia, 13-16 de junio de 2006), pp. 350-354. Disponible en: https//researchspace.auckland. ac.nz/bitstream/handle/2292/20329/lifelongConf\%202006Paper 37-final.pdf?sequence $=6$ [Fecha de consulta: 27/12/2012] .

Wang, L. (2007), "Sociocultural learning theories and information literacy teaching activities in higher education", en Reference $\mathcal{E}$ User Services Quarterly, vol. 47, núm 2, pp. 149-158.

-(2011), "An information literacy integration model and its application in higher education", en Reference Services Review, vol. 39, núm. 4, pp. 703-720.

-; Bruce, C. y Hughes, H. (2011), "Sociocultural theories and their application in information literacy research and education", en Australian Academic E Research Libraries, vol. 42, núm. 4, pp. 296-308.

Wang, Y. (2007), "Riding to the future: An investigation of information literacy skills of students at an urban university as applied to the web environment", en International Journal on E-Learning, vol. 6, núm. 4, pp. 593-603.

Winterman, B.; Donovan, C. y Slough, R. (2011), "Literacy for multiple disciplines: Toward a campus-wide integration model at Indiana University, Bloomington", en Communication in Information Literacy, vol. 5, núm. 1, pp. 38-54.

Yua, H.; Arpah, S.; Awang, S. y Abrizahb, A. (2011), "Integrating information literacy instruction (ILI) through resource-based school projects: An interpretive exploration", en Education for Information, vol. 28, núm. 2, pp. 247-268.

Zapata-Ros, M. (2012), "Calidad en entornos ubicuos de aprendizaje”, en Revista de Educación a Distancia, núm. 31. Disponible en: www. um.es/ead/red/31/zapata_ros.pdf [Fecha de consulta: 20/08/2012]. 
Descripción general de los artículos seleccionados

\begin{tabular}{|c|c|c|c|c|}
\hline & Título & Año & País / Universidad & Tipo de estudio /Categoría \\
\hline 1 & $\begin{array}{l}\text { Sociocultural learning theories } \\
\text { and information literacy } \\
\text { teaching activities in higher } \\
\text { education }\end{array}$ & 2007 & $\begin{array}{l}\text { Nueva Zelanda/Universidad } \\
\text { de Auckland }\end{array}$ & $\begin{array}{l}\text { Experiencia/Enfoque y } \\
\text { Modelo }\end{array}$ \\
\hline 2 & $\begin{array}{l}\text { Riding to the future: An inves- } \\
\text { tigation of information literacy } \\
\text { skills of students at an urban } \\
\text { university as applied to the web } \\
\text { environment }\end{array}$ & 2007 & $\begin{array}{l}\text { Estados Unidos/Universidad } \\
\text { de Alabama }\end{array}$ & $\begin{array}{l}\text { Investigación/Enfoque y } \\
\text { Modelo }\end{array}$ \\
\hline 3 & $\begin{array}{l}\text { La alfabetización en Informa- } \\
\text { ción: un primer paso en la } \\
\text { formación de los Bibliotecólo- } \\
\text { gos en la Universidad de Playa } \\
\text { Ancha }\end{array}$ & 2007 & $\begin{array}{l}\text { Chile/Universidad Tecnológica } \\
\text { Metropolitana }\end{array}$ & $\begin{array}{l}\text { Investigación/Enfoque, } \\
\text { Modelo y Estrategias }\end{array}$ \\
\hline 4 & $\begin{array}{l}\text { El desarrollo de competencias } \\
\text { informativas en estudiantes } \\
\text { universitarios a través de un } \\
\text { curso con valor en créditos }\end{array}$ & 2007 & $\begin{array}{l}\text { México/Universidad Autóno- } \\
\text { ma de Ciudad Juárez }\end{array}$ & $\begin{array}{l}\text { Experiencia/Enfoque, } \\
\text { Modelo y Estrategias }\end{array}$ \\
\hline 5 & $\begin{array}{l}\text { Diseño de un curso en línea } \\
\text { para la alfabetización informa- } \\
\text { cional bajo el modelo ADDIE: } \\
\text { una experiencia en la UNAM }\end{array}$ & 2007 & $\begin{array}{l}\text { México/Universidad Nacional } \\
\text { Autónoma de México }\end{array}$ & $\begin{array}{l}\text { Experiencia/Enfoque y } \\
\text { Estrategias }\end{array}$ \\
\hline 6 & $\begin{array}{l}\text { La biblioteca digital y la edu- } \\
\text { cación a distancia como entes } \\
\text { inseparables para incrementar } \\
\text { la calidad de la educación }\end{array}$ & 2008 & $\begin{array}{l}\text { México/Universidad Nacional } \\
\text { Autónoma de México }\end{array}$ & $\begin{array}{l}\text { Investigación/Enfoque y } \\
\text { Estrategias }\end{array}$ \\
\hline 7 & $\begin{array}{l}\text { Using writing assignments to } \\
\text { improve self-assessment and } \\
\text { communication skills in an } \\
\text { engineering statics course }\end{array}$ & 2008 & $\begin{array}{l}\text { Estados Unidos/Instituto de } \\
\text { Tecnología Rose-Hulman }\end{array}$ & $\begin{array}{l}\text { Investigación/Enfoque y } \\
\text { Estrategias }\end{array}$ \\
\hline 8 & $\begin{array}{l}\text { La formación en competencias } \\
\text { informáticas e informacionales } \\
\text { desde la Escuela Interameri- } \\
\text { cana de Bibliotecología con } \\
\text { el apoyo de una plataforma } \\
\text { de e-learning. Experiencias y } \\
\text { resultados }\end{array}$ & 2010 & $\begin{array}{l}\text { Colombia/Universidad de } \\
\text { Antioquia }\end{array}$ & $\begin{array}{l}\text { Experiencia/Enfoque, } \\
\text { Modelo y Estrategias }\end{array}$ \\
\hline 9 & $\begin{array}{l}\text { Formarnos y autoformarnos en } \\
\text { alfabetización informacional. Un } \\
\text { programa de mentorización en } \\
\text { bibliotecas universitarias-CRAI }\end{array}$ & 2010 & $\begin{array}{l}\text { España/Universidad de } \\
\text { Granada }\end{array}$ & $\begin{array}{l}\text { Experiencia/Enfoque y } \\
\text { Estrategias }\end{array}$ \\
\hline 10 & \begin{tabular}{|l|} 
Un plan de formación en \\
competencias de información \\
a través de aulas virtuales: \\
análisis de una experiencia con \\
alumnado universitario
\end{tabular} & 2010 & $\begin{array}{l}\text { España/Universidad de la } \\
\text { Laguna }\end{array}$ & $\begin{array}{l}\text { Experiencia/Enfoque, } \\
\text { Modelo y Estrategias }\end{array}$ \\
\hline
\end{tabular}




\begin{tabular}{|c|c|c|c|c|}
\hline 11 & $\begin{array}{l}\text { Investigating the Effect of } \\
\text { Internet Information Literacy on } \\
\text { the Development of University } \\
\text { Students' Metacognitive Skills }\end{array}$ & 2011 & $\begin{array}{l}\text { Irán/Universidad Islámica } \\
\text { Azad }\end{array}$ & $\begin{array}{l}\text { Investigación/Enfoque y } \\
\text { Estrategias }\end{array}$ \\
\hline 12 & $\begin{array}{l}\text { Sociocultural theories and } \\
\text { their application in information } \\
\text { literacy research and education }\end{array}$ & 2011 & $\begin{array}{l}\text { Nueva Zelanda/Universidad } \\
\text { Tecnológica de Quensland }\end{array}$ & Experiencia/Enfoque \\
\hline 13 & $\begin{array}{l}\text { ¿Cómo desarrollar habilidades } \\
\text { para la obtención de informa- } \\
\text { ción científica? Una propuesta } \\
\text { desde la clase de Física }\end{array}$ & 2012 & $\begin{array}{l}\text { Cuba/Universidad de Ciencias } \\
\text { Pedagógicas }\end{array}$ & $\begin{array}{l}\text { Experiencia/Enfoque y } \\
\text { Estrategias }\end{array}$ \\
\hline 14 & $\begin{array}{l}\text { Efficacy of Integrating Infor- } \\
\text { mation Literacy Education Into } \\
\text { a Women's Health Course on } \\
\text { Information Literacy for RN- } \\
\text { BSN Students }\end{array}$ & 2007 & Taiwán/Universidad de Fooyin & Investigación/Modelo \\
\hline 15 & $\begin{array}{l}\text { An approach to curriculum } \\
\text { for improving the Information } \\
\text { literacy Skills and academic } \\
\text { Writing of Students in the Field } \\
\text { of nursery in Hong Kong }\end{array}$ & 2008 & $\begin{array}{l}\text { Estados Unidos y China/ } \\
\text { Universidad de Hawaii, Univer- } \\
\text { sidad de Hong Kong }\end{array}$ & Experiencia/Modelo \\
\hline 16 & $\begin{array}{l}\text { Integrating STEM Information } \\
\text { Competencies into an Under- } \\
\text { graduate Curriculum }\end{array}$ & 2010 & $\begin{array}{l}\text { Estados Unidos/Universi- } \\
\text { dad Politécnica Estatal de } \\
\text { California }\end{array}$ & Experiencia/Modelo \\
\hline 17 & $\begin{array}{l}\text { La evaluación de los programas } \\
\text { de alfabetización en informa- } \\
\text { ción en la educación superior: } \\
\text { estrategias e instrumentos }\end{array}$ & 2010 & $\begin{array}{l}\text { España/Universidad Carlos III } \\
\text { de Madrid }\end{array}$ & Experiencia/Modelo \\
\hline 18 & $\begin{array}{l}\text { Research is a verb: Exploring a } \\
\text { new information literacy-embe- } \\
\text { dded undergraduate research } \\
\text { methods course }\end{array}$ & 2010 & $\begin{array}{l}\text { Canadá/Universidad de } \\
\text { Alberta }\end{array}$ & Experiencia/Modelo \\
\hline 19 & $\begin{array}{l}\text { Evaluation of a university onli- } \\
\text { ne. Information Literacy unit }\end{array}$ & 2010 & $\begin{array}{l}\text { Australia/Universidad del } \\
\text { Oeste de Australia }\end{array}$ & Experiencia/Modelo \\
\hline 20 & $\begin{array}{l}\text { Information Literacy Training } \\
\text { for Postgraduate and Postdoc- } \\
\text { toral Researchers: a National } \\
\text { Survey and its Implications }\end{array}$ & 2010 & $\begin{array}{l}\text { Reino Unido/Leeds University } \\
\text { Business School }\end{array}$ & Investigación/Modelo \\
\hline 21 & $\begin{array}{l}\text { Information literacy education } \\
\text { and instruction in academic } \\
\text { libraries and LIS schools in } \\
\text { institutions of higher education } \\
\text { in South Africa }\end{array}$ & 2010 & $\begin{array}{l}\text { Sur África/Universidad de Sur } \\
\text { África }\end{array}$ & Investigación/Modelo \\
\hline 22 & $\begin{array}{l}\text { Is an online learning module } \\
\text { an effective way to develop } \\
\text { information literacy skills? }\end{array}$ & 2010 & $\begin{array}{l}\text { James Cook University/ } \\
\text { Australia }\end{array}$ & Experiencia/Modelo \\
\hline 23 & $\begin{array}{l}\text { Assessing information literacy } \\
\text { competency of Information } \\
\text { Science and Library Manage- } \\
\text { ment graduate students of }\end{array}$ & 2010 & $\begin{array}{l}\text { Bangladeshi/Universidad de } \\
\text { Dhaka }\end{array}$ & Investigación/Modelo \\
\hline
\end{tabular}




\begin{tabular}{|c|c|c|c|c|}
\hline & $\begin{array}{l}\text { Dhaka University Mohammed } \\
\text { Anwarul Islam University of } \\
\text { Dhaka }\end{array}$ & & & \\
\hline 24 & $\begin{array}{l}\text { La alfabetización en informa- } \\
\text { ción en los Policlínicos Univer- } \\
\text { sitarios }\end{array}$ & 2011 & $\begin{array}{l}\text { Cuba/Policlínico Universitario } \\
19 \text { de Abril }\end{array}$ & $\begin{array}{l}\text { Experiencia/Modelo y } \\
\text { Estrategias }\end{array}$ \\
\hline 25 & $\begin{array}{l}\text { Alfinev. Modelo para evaluar la } \\
\text { alfabetización informacional en } \\
\text { la educación superior en Cuba }\end{array}$ & 2011 & $\begin{array}{l}\text { Cuba/Universidad Central } \\
\text { "Marta Abreu" de Las Villas }\end{array}$ & Investigación/Modelo \\
\hline 26 & $\begin{array}{l}\text { Mapping the journey: Developing } \\
\text { an information literacy strategy } \\
\text { as part of curriculum reform }\end{array}$ & 2011 & $\begin{array}{l}\text { Australia/Universidad de La } \\
\text { Trobe }\end{array}$ & Experiencia/Modelo \\
\hline 27 & $\begin{array}{l}\text { Literacy for multiple disciplines: } \\
\text { Toward a campus-wide } \\
\text { integration model at Indiana } \\
\text { University, Bloomington }\end{array}$ & 2011 & $\begin{array}{l}\text { Estados Unidos/Universidad } \\
\text { Indiana, Universidad de } \\
\text { Wisconsin }\end{array}$ & Investigación/Modelo \\
\hline 28 & $\begin{array}{l}\text { Entering unfamiliar territory } \\
\text { building an information literacy } \\
\text { course for graduate students in } \\
\text { interdisciplinary areas }\end{array}$ & 2011 & $\begin{array}{l}\text { Estados Unidos/Universidad } \\
\text { de Kentucky }\end{array}$ & Experiencia/Modelo \\
\hline 29 & $\begin{array}{l}\text { Learning Outcomes of Infor- } \\
\text { mation Literacy Instruction at } \\
\text { Business Schools }\end{array}$ & 2011 & $\begin{array}{l}\text { Canadá/Universidad de Mc- } \\
\text { Master, Universidad de Alberta, } \\
\text { Universidad de Mount Royal, } \\
\text { Universidad de Lakehead }\end{array}$ & Investigación/Modelo \\
\hline 30 & $\begin{array}{l}\text { La alfabetización informacional } \\
\text { en la educación superior: una } \\
\text { mirada desde el análisis crítico } \\
\text { del discurso }\end{array}$ & 2013 & $\begin{array}{l}\text { Colombia/Universidad Católi- } \\
\text { ca del Norte }\end{array}$ & $\begin{array}{l}\text { Investigación/Modelo y } \\
\text { Estrategias }\end{array}$ \\
\hline 31 & $\begin{array}{l}\text { Propuesta de competencia } \\
\text { en gestión de información y } \\
\text { niveles de desempeño para la } \\
\text { docencia universitaria }\end{array}$ & 2008 & Chile/Universidad de Talca & Investigación/Estrategias \\
\hline 32 & $\begin{array}{l}\text { ¿Cómo manejan información } \\
\text { los estudiantes de educación } \\
\text { superior? El caso de la Univer- } \\
\text { sidad de La Sabana, Colombia }\end{array}$ & 2011 & $\begin{array}{l}\text { Colombia/Universidad de la } \\
\text { Sabana }\end{array}$ & Investigación/Estrategias \\
\hline 33 & $\begin{array}{l}\text { Alfabetización informacional en } \\
\text { la educación superior virtual: } \\
\text { logros y desafíos }\end{array}$ & 2012 & $\begin{array}{l}\text { Colombia/Universidad de la } \\
\text { Sabana }\end{array}$ & Investigación/Estrategias \\
\hline 34 & $\begin{array}{l}\text { Taxonomy of adolescent meta- } \\
\text { cognitive knowledge during the } \\
\text { information search process }\end{array}$ & 2010 & $\begin{array}{l}\text { Estados Unidos/Universidad } \\
\text { de Pittsburgh }\end{array}$ & Investigación/Estrategias \\
\hline 35 & $\begin{array}{l}\text { The effect of the use of learning } \\
\text { journals on the development of } \\
\text { metacognition in undergradua- } \\
\text { te students }\end{array}$ & 2009 & $\begin{array}{l}\text { Estados Unidos/Capella } \\
\text { University }\end{array}$ & Investigación/Estrategias \\
\hline 36 & $\begin{array}{l}\text { Integrating information literacy } \\
\text { instruction (ILI) through resour- } \\
\text { ce-based school projects: An } \\
\text { interpretive exploration }\end{array}$ & 2010 & $\begin{array}{l}\text { Malasia/University Teknologi } \\
\text { MARA, Universidad de Malaya }\end{array}$ & Investigación/Estrategias \\
\hline
\end{tabular}




\begin{tabular}{|c|c|c|c|c|}
\hline 37 & $\begin{array}{l}\text { The Search for the Skunk Ape: } \\
\text { Studying the Impact of an Onli- } \\
\text { ne Information Literacy Tutorial } \\
\text { on Student Writing }\end{array}$ & 2011 & $\begin{array}{l}\text { Estados Unidos/Universidad } \\
\text { de Georgia Southern, Universi- } \\
\text { dad de Florida Gulf Coast }\end{array}$ & Investigación/Estrategias \\
\hline 38 & $\begin{array}{l}\text { Taking Library } 2.0 \text { to the Next } \\
\text { Level: Using a Course Wiki for } \\
\text { Teaching Information Literacy } \\
\text { to Honors Students }\end{array}$ & 2010 & $\begin{array}{l}\text { Estados Unidos, Universidad } \\
\text { de Rhode Island }\end{array}$ & Experiencia/Estrategias \\
\hline 39 & $\begin{array}{l}\text { Information Literacy Follow- } \\
\text { Through: Enhancing Preservice } \\
\text { Teachers' Information Evalua- } \\
\text { tion Skills Through Formative } \\
\text { Assessment }\end{array}$ & 2011 & $\begin{array}{l}\text { Estados Unidos/Universidad } \\
\text { Estatal de Boise }\end{array}$ & Investigación/Estrategias \\
\hline 40 & $\begin{array}{l}\text { Formative and summative } \\
\text { assessment }\end{array}$ & 2009 & $\begin{array}{l}\text { Estados Unidos/Universidad } \\
\text { Estatal de Boise }\end{array}$ & Investigación/Estrategias \\
\hline 41 & $\begin{array}{l}\text { Learning Through Quests and } \\
\text { Contests: Games in Information } \\
\text { Literacy Instruction }\end{array}$ & 2011 & $\begin{array}{l}\text { Estados Unidos/New York City } \\
\text { College of Technology, CUNY }\end{array}$ & Experiencia/Estrategias \\
\hline 42 & $\begin{array}{l}\text { Quarantined: AxI Wise and the } \\
\text { Information Outbreak: Creating } \\
\text { an online game to teach infor- } \\
\text { mation literacy skills }\end{array}$ & 2007 & $\begin{array}{l}\text { Estados Unidos/Universidad } \\
\text { Estatal de Arizona }\end{array}$ & Experiencia/Estrategias \\
\hline 43 & $\begin{array}{l}\text { Will undergraduate students } \\
\text { play games to learn how to } \\
\text { conduct library research? }\end{array}$ & 2009 & $\begin{array}{l}\text { Estados Unidos/Universidad } \\
\text { de Michigan }\end{array}$ & Investigación/Estrategias \\
\hline 44 & $\begin{array}{l}\text { Games for teaching information } \\
\text { literacy skills }\end{array}$ & 2007 & $\begin{array}{l}\text { Estados Unidos/Universidad } \\
\text { de Notre Dame }\end{array}$ & Experiencia/Estrategias \\
\hline 45 & $\begin{array}{l}\text { Testing Information Literacy } \\
\text { in Digital Environments: ETS's } \\
\text { iSkills Assessment }\end{array}$ & 2007 & Estados Unidos/ETS & Experiencia/Estrategias \\
\hline 46 & $\begin{array}{l}\text { Unir Educación y Bibliotecas: } \\
\text { La evolución de un reto } \\
\text { permanente }\end{array}$ & 2013 & España/Universidad de Murcia & Investigación/Estrategias \\
\hline 47 & $\begin{array}{l}\text { Recolectores, verificadores } \\
\text { y reflexivos: perfiles de la } \\
\text { competencia informacional en } \\
\text { estudiantes universitarios de } \\
\text { primer semestre }\end{array}$ & 2010 & $\begin{array}{l}\text { Colombia/Pontificia Universi- } \\
\text { dad Javeriana y Universidad } \\
\text { Industrial de Santander }\end{array}$ & Investigación/Modelo \\
\hline 48 & $\begin{array}{l}\text { Reconceptualización sobre las } \\
\text { competencias Informacionales. } \\
\text { Una experiencia en la Educa- } \\
\text { ción Superior }\end{array}$ & 2010 & $\begin{array}{l}\text { Colombia/Universidad Indus- } \\
\text { trial de Santander }\end{array}$ & Investigación/Modelo \\
\hline 49 & $\begin{array}{l}\text { Dimensiones socioculturales de } \\
\text { la competencia informacional } \\
\text { en estudiantes universitarios: } \\
\text { creencias, cultura y experien- } \\
\text { cias vitales }\end{array}$ & 2011 & $\begin{array}{l}\text { Colombia/Pontificia Universi- } \\
\text { dad Javeriana }\end{array}$ & Investigación/Modelo \\
\hline
\end{tabular}




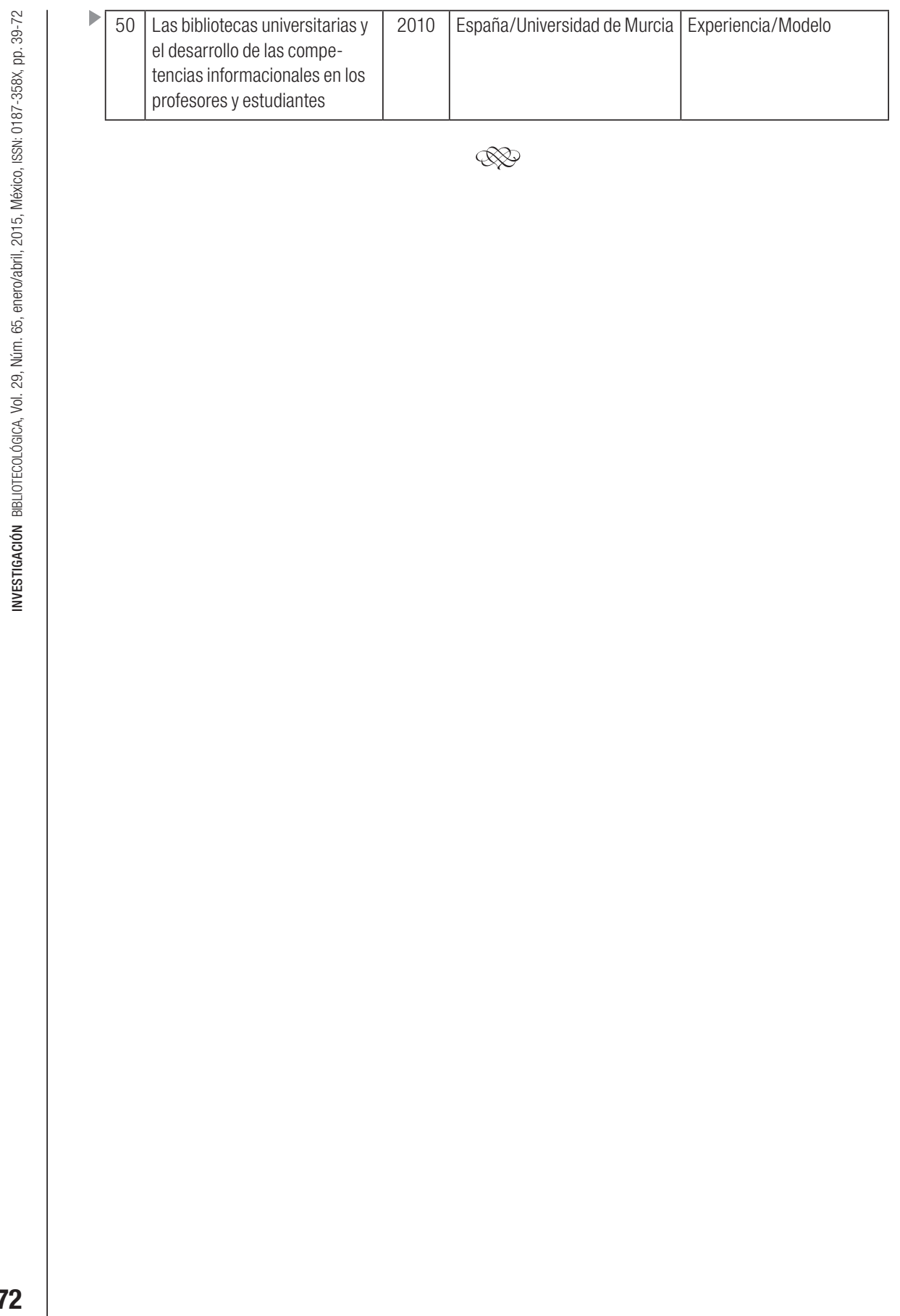

